\title{
Recurrent bacterial symptomatic cystitis: A pilot study on a new natural option for treatment
}

\author{
Giulio Del Popolo, Federico Nelli \\ Department of Neuro-Urology, Azienda Ospedaliero-Universitaria Careggi, Firenze, Italy.
}

\begin{abstract}
Summary Objectives: The aim of our study was to explore the effectiveness of the combination of D-mannose, Salicin, and Lactobacillus acidophilus (La-14) in patients complaining recurrent symptomatic cystitis due to E. coli.

Materials and methods: From July 2013 to September 2014, 85 consecutive subjects (68 women and 17 men) affected by recurrent symptomatic cystitis were enrolled. Of those, 46 (33 women and 13 men) suffered from neurogenic bladder. Overall 78 patients received an initial 5-days regimen consisting on a tid oral combination of $1000 \mathrm{mg}$ of D-mannose plus $200 \mathrm{mg}$ of dry willow extract (salicin) (attack phase), followed by bid 7-days with $700 \mathrm{mg}$ of D-mannose plus $50 \mathrm{mg}\left(1 \times 10^{9}\right.$ CFU) of Lactobacillus acidophilus (La-14) (maintenance treatment). The maintenance treatment was repeated every 15 days for the next two months. Patients' symptoms were evaluated through a 3-days bladder diary and a Visual Analogic Scale (VAS).

Results: After treatment VAS scores decreased from $8.07 \pm$ 1.70 to $4.74 \pm 2.07$ ( $p=0.001)$ in non-neurological patients (group A) and from $7.21 \pm 1.90$ to $3.74 \pm 3.12(p=0.001)$ in the neurological patients (group B). A significant reduction of daily frequency was noted in both groups: from $14 \pm 3$ to $7 \pm$ $3(p=0.001)$ in group $A$ and from $15 \pm 3$ to $8 \pm 3(p=0.001)$ in group $B$. A reduction of incontinence episodes in Group $A$ patients was observed, as well as in 12/39 Group B.

Improvements were maintained during follow- up.

Conclusion: This therapeutic approach combining D-Mannose with Salicin (acute treatment) and Lactobacillus acidophilus La-14 (maintaining treatment) seems to be effective in symptomatic bacterial UTIs. Further larger and randomized control trials (RCTs) are needed to confirm our results.
\end{abstract}

KEY WORDS: Bacterial cystitis; UTI; D-mannose; Neurogenic bladder dysfunction, Lactobacillus acidophilus La-14; Salicin.

Submitted 19 March 2018; Accepted 9 May 2018

\section{INTRODUCTION}

Urinary tract infections (UTIs) are among the most common infections in humans and affect both sexes and all age groups. Among them, cystitis is a clinical syndrome characterized by dysuria, urinary frequency and urgency, with or without suprapubic pain. The most common cause of cystitis is bacterial from common pathogens of the lower urinary tract. Bacterial cystitis is usually associated with bacteriuria and leukocyturia.

In Europe, Escherichia coli (E. coli) is the most common uropathogen, accounting for up to $86 \%$ of all UTIs (1); other pathogens are Proteus and Klebsiella species, enterococci, Group B streptococci and Pseudomonas aeruginosa, but UTIs can be also due to the association of several pathogens with greater clinical impact and potential complications.

Women are more likely to be affected by UTI than men, with an estimated incidence of 142 in 3 among females versus only 1 in 20 among males (14). The higher incidence of UTIs among women can be attributed to the shorter urethral length, which provides an effective barrier against bacterial contamination.

UTIs can also be due to neurogenic bladder and it may result from decreased local immune defenses, impaired lower urinary tract function, bladder management (e.g. intermittent catheterism, indwelling catheter) and the coexisting condition of neurogenic bowel dysfunction. In any case an excessive use of antibiotics may also increase the UTI risk by altering the normal microbiota and increasing bacterial resistance.

Treatment is generally multimodal and includes antibiotic and anti-inflammatory therapy together with dietary supplements and behavioral interventions.

Recommended preventive measures such as adequate water intake, rhythm in urinary voiding and non-antibiotic prophylaxis (e.g., cranberry, urine acidifiers, probiotics) are not yet supported by evidence $(3,4)$.

D-mannose has long been studied and has been shown to be effective and safe in treating and preventing UTIs also in neurological populations such as multiple sclerosis (2). Through its mechanism of action, it prevents bacterial colonization and invasion of the bladder by pathogens, and is effective in multiresistant UPEC (Uropathogenic E. coli) infections (5). In vitro studies have shown that D-mannose binds and blocks FimH adhesins positioned on the tip of type 1 bacterial fimbriae.

During bacterial colonization, FimH binds to the glycoprotein-containing carbohydrate receptors on the urinary epithelium. D-mannose acts as a competitive inhibitor of bacterial adhesion as it has a structure similar to the binding site of glycoproteic urothelial receptors; in sufficient concentrations in urine, D-mannose causes saturation of FimH adhesins and therefore prevents bacteria from binding to urothelial receptors.

In vitro studies show that the local application of D-mannose decreased the adherence of E. coli, Pseudomonas aeruginosa and Streptococcus zooepidemicus to urothelial endometrial cells in horses. Reduction of bacteriuria lev-

\footnotetext{
No conflict of interest declared.
} 
els has also been confirmed by in vivo animal models of recurrent UTIs. A randomized, non-blind, placebo-controlled study to evaluate prophylaxis of recurrent urinary infections conducted on 308 women showed that Dmannose used for 6 months at a dose of $2 \mathrm{~g}$ per day was significantly more effective than placebo and as effective as nitrofurantoin $50 \mathrm{mg}$ in preventing recurrence $(6,7)$. Salicin (an alcoholic $\beta$-glucoside between glucose and salicylic alcohol), has anti-inflammatory properties (8). Lactobacilli possess antimicrobial properties that regulate the urogenital microbiota. Lactobacillus acidophilus La-14 has shown excellent adherence to epithelial surfaces in vitro demonstrating inhibition of selected pathogens, including E. coli (9-12).

The recurrence of urogenital infections leads to a change in local flora from a predominance of lactobacilli to coliform uropathogens.

Therefore, the use of systemic probiotics containing Lactobacillus acidophilus that help to restore the microbiota has been proposed for the treatment and prophylaxis of bacterial urogenital infections (13).

The aim of the present clinical study was to evaluate the feasibility and efficacy of a new combined 2-phase approach in order to treat neurological and non-neurological patients affected by recurrent symptomatic bacterial cystitis mainly due to E. coli.

\section{MATERIALS AND METHOdS}

Between July 2013 and September 2014, consecutive subjects attending to our Neuro-Urology clinic with diagnosis of recurrent bacterial cystitis, were enrolled in our study. Definition of symptomatic UTIs included the presence of at least 2 of the following symptoms: dysuria, new onset and/or worsening of urgency and/or urgency urinary incontinence, flank and/or suprapubic pain, worsening of muscle spasticity (in SCI and MS patients) and/or autonomic dysreflexia (in SCI patients) combined with $\mathrm{a} \geq 10^{5}$ $\mathrm{CFU} / \mathrm{mL}$ at urine culture (in non-neurological) or $\geq 10^{6}$ $\mathrm{CFU} / \mathrm{mL}$ (in neurological population). Only adults between 18 and 80 years-old were included. Other inclusion criteria were: history of $\geq 3$ acute episodes of recurrent symptomatic cystitis in the last year with at least two positive urine cultures for E. coli in the last 3 months before the screening, previous unsuccessful treatment with D-mannose and/or cranberry. Exclusion criteria was the presence of positive urine culture not due to E. coli at the screening, pregnancy, breastfeeding, haematuria, fever, any uro-genital abnormalities at ultrasound, history of any allergies to salicylates and/or D-mannose, any use of antiobiotics for any reasons within the last two weeks before the enrollment and overall during the study.

All patients received an initial 5-days regimen consisting on an oral combination of $1000 \mathrm{mg}$ of D-mannose plus $200 \mathrm{mg}$ of dry willow extract (salicin) three times daily (attack phase, morning-midday-evening always on a full stomach), followed by 7-days with $700 \mathrm{mg}$ of D-mannose plus $50 \mathrm{mg}\left(1 \times 10^{9} \mathrm{CFU}\right)$ of Lactobacillus acidophilus (La-14) twice daily (maintenance treatment, morning and evening on a full stomach). This latest combination (D-mannose plus La-14) was repeated at the same dosage for 15-days at each month for two months.
Changes in patients' symptoms was monitored at baseline (T0), 2-weeks (T1), 12-weeks (end of treatment, T2) and at one month after the end of treatment (T3) by a 3-days bladder diary and the Visual Analogic Scale (VAS). The efficacy in preventing new recurrences was assessed by repeated urine cultures during follow-ups.

\section{RESULTS}

Eighty-five patients were included in our study with a mean age of 45,2 years (range, 22-78). Forty-six out of 85 were affected by neurogenic bladder (Group B) due to spinal cord injury or multiple sclerosis ( 33 females and 13 males) with a mean age of 37,4 years (range, 25-54). Thirty-seven out of 46 neurological patients were managed by intermittent catheterization regimen. Seven patients in the Group B missed the T2 follow up, then were considered as drop out. None of the 39 non-neurological patients (group A) dropped out during the study. Patients with neurogenic bladder (group B) reported a decrease of mean VAS scores from $7.21 \pm 1.90$ to $3.74 \pm$ 3.12 starting from the first days of treatment until the end of the attack and maintenance phases (T1), and maintained the result at $\mathrm{T} 2$ and one month after the end of treatment (T3). Symptoms at baseline were pain, urgency/frequency, urinary incontinence (Table 1). Again, a decrease of the mean VAS scores during followup was also found in group A. This result was maintained at T2 and T3. The improvements in clinical symptoms in both groups (e.g. dysuria, frequency, urgency) were already significant 2 weeks after (T1) the beginning of treatment and these results were confirmed after the maintenance therapy (T2) and 1 month after the end of treatment (T3) (Table 2).

In particular, group A showed a significant decrease from $14.0 \pm 2.6$ to $6.9 \pm 1.3(\mathrm{p}=0.001)$ in daily urinary frequency at $\mathrm{T} 1$, a reduction that was maintained both at $\mathrm{T} 2$ $(6.5 \pm 1.2)$ and one month after the end of treatment (T3) $6.2 \pm 0.9$

In Group B, a significant reduction in daily urinary frequency from $15 \pm 3$ to $8 \pm 3(p=0.001)$ was observed at $\mathrm{T} 1$, a reduction that was maintained both at $\mathrm{T} 2$ and one month after the end of treatment (T3).

\section{Table 1.}

Symptoms at baseline.

\begin{tabular}{|lcc|}
\hline & Group $\mathbf{A}$ & Group B \\
\hline Pain $>3$ on VAS scale & Pts $n^{\circ}$ & Pts $n^{\circ}$ \\
\hline Urgency/frequency $\geq 8$ times/day & $33 / 39$ & $15 / 39$ \\
\hline Incontinence episodes & $39 / 39$ & $26 / 39$ \\
\hline
\end{tabular}

Table 2.

Mean VAS score pre and during treatment in both population.

\begin{tabular}{|lcc|}
\hline & Group A & Group B \\
\hline VAS Score T0 & $8.07( \pm 1.70)$ & $7.21( \pm 1.9)$ \\
\hline VAS Score T1 & $4.74( \pm 2.07)$ & $3.74( \pm 3.12)$ \\
\hline VAS Score T2 & $4.45( \pm 2.19)$ & $3.51( \pm 2.32)$ \\
\hline VAS Score T3 & $4.24( \pm 2.23)$ & $3.40( \pm 2.20)$ \\
\hline
\end{tabular}


In this Group B we observed also a reduction of the incontinence episodes at the 3-days bladder diary in 11 patients managed by intermittent catheterization ranging from 4.2+/- 0.60 (T0) to $2.8+/-1,33$ (T2) (p > 0.001).

No significant side effect was reported during the treatment

\section{Discussion}

Urinary tract infections are still a major problem in clinical practice. Advances in antibiotic therapy have allowed clinicians to decrease the duration of treatment and drastically reduce the complications associated with UTIs but they have not reduced UTI frequency. On the other hand, an indefinite antibiotic prophylaxis would lead to selection of multiresistant microbial strains and exposure of the patients to serious infectious complications.

Various publications emphasize the importance of a moderate use of antibiotics. For this reason, research has focused on enhancing exogenous antibacterial defenses in the bladder.

An attack treatment with D-mannose and salicin, followed by maintenance and/or prophylaxis therapy with D-mannose and probiotics was found to be effective in the treatment of urological infections, including recurrent bacterial cystitis (predominantly due to E. coli) also in different populations (neurological vs non-neurological) D-mannose acts to promote the elimination of bacteria from the bladder and Lactobacillus acidophilus helps to rebalance the intestinal flora, thus reducing the presence of E. coli, which could reach the bladder via the urethra.

The administration of D-mannose in combination with salicin results in an additive anti- inflammatory activity of the two components in the treatment of inflammation and pain In addition, D-mannose and probiotics, administered in combination rather than separately, have demonstrated a surprising complementary activity resulting in a bacteria-repelling effect, which, unlike bactericidal or bacteriostatic effects, allows removal of the pathogenic bacteria/bacilli. Although statistics was limited due to the small number and heterogeneity of patients, the study shows that this combination may be effective in resolving sympoms of UTI. In addition, with the use of this proposed treatment we avoided to repeat antibiotics even in case of E. coli positivity during follow-up because of early solved acute symptoms at the first evaluation which was maintained also one month after the end of treatment. We are conscious that the main limit of this single-arm study was the lack of placebo-control. Again no comparisons with other possible treatment combinations or single use of any of these solutions was done, although we intentionally included patients who were already unsuccessfully treated with D-mannose and/or cranberry before study.

\section{Conclusions}

In our experience we found that the administration of an initial combination of D-mannose and salicin and a maintenance and/or prophylactic combination of Dmannose and probiotics, in particular Lactobacillus acidophilus, could be effective in the treatment and prophy- laxis of recurrent cystitis with E. coli infection both in non-neurological and neurological patients.

The treatment effectiveness can be measured with the resolution of cystitis-related symptoms.

This combination treatment could be also promising as adjuvant therapy due to its bacteria-repelling, antiinflammatory and analgesic properties and rebalancing effects on the intestinal flora, leading to reduced antibiotic use and resistance.

These positive results, obtained with these treatment and schedule, should be validated by further larger and randomized studies.

\section{REFERENCES}

1. Flores-Mireles AL, Walker JN, Caparon M, Hultgren SJ. Urinary tract infections: epidemiology, mechanisms of infection and treatment options. Nat Rev Microbiol 2015; 13:269-284.

2. Phé V, Pakzad M, Haslam C, et al. Open label feasibility study evaluating D-mannose combined with home- based monitoring of suspected urinary tract infections in patients with multiple sclerosis. Neurourol Urodyn. 2017; 36:1770-1775.

3. Jepson RG, Williams G, Craig JC. Cranberries for preventing urinary tract infections. Cochrane Database Syst Rev. 2012; 10:CD001321

4. Juthani-Mehta M, Van Ness PH, Bianco L, et al. Effect of cranberry capsules on bacteriuria plus pyuria among older women in nursing homes. JAMA. 2016; 316:1879-1887.

5. Hickling DR, Nitti VW. Management of recurrent urinary tract infections in healthy adult women. Rev Urol. 2013; 15:41-48.

6. Kranjčec B, Papeš D, Altarac S. D-mannose powder for prophylaxis of recurrent urinary tract infections in women: a randomized clinical trial. World J Urol. 2014; 32:79-84.

7. Altarac S, Papes D. Use of d-mannose in prophylaxis of recurrent urinary tract infections (UTIS) in women. BJUI. 2014; 9-10.

8. Khayyal MT, El-Ghazaly MA, Abdallah DM, et al. Mechanisms involved in the anti-inflammatory effect of a standardized willow bark extract. Arzneimittelforschung. 2005; 55:677-87.

9. Shim YH, Lee SJ, Lee JW. Antimicrobial activity of lactobacillus strains against uropathogens. Pediatr Int. 2016; 58:1009-1013.

10. Ibrahem KH, Hasan S. Studying the ability of lactobacillus bacteria inhibit the growth of uropathogens and their adhesion to uroepithelial cells. J College of Basic Education. 2009; 60:73-84.

11. Todorov SD, Furtado DN, Saad SM, et al. Bacteriocin production and resistance to drugs are advantageous features for Lactobacillus acidophilus La-14, a potential probiotic strain. New Microbiol. 2011; 34:357-70.

12. Lactobacillus acidophilus La-14. Technical Memorandum. Danisco.

13. Barrons R, Tassone D. Use of Lactobacillus probiotics for bacterial genitourinary infections in women: a review. Clin Ther. 2008; 30:453-6.

14. Foxman B, Barlow R, D'Arcy $H$, et al. Urinary tract infection: self-reported incidence and associated costs. Ann Epidemiol. 2000; 10:509-515.

\section{Correspondence}

Giulio Del Popolo, MD - dpgiulio@gmail.com

Federico Nelli, MD

Department of Neuro-Urology, Azienda Ospedaliero-Universitaria Careggi L.go G. Alessandro Brambilla, 3 - Firenze, Italy 